\title{
Iron excretion in urine in patients with acute kidney injury after cardiac surgery
}

\author{
Jowita Biernawska ${ }^{1, A-F}$, Joanna Bober ${ }^{2, C}$, Katarzyna Kotfis ${ }^{3, A, B, D}$, Iwona Noceńn $2, B$, \\ Anna Bogacka ${ }^{4, B}$, Edyta Barnik ${ }^{3, D}$, Dariusz Chlubek ${ }^{5, C}$, Maciej Żukowski ${ }^{3, A, C, E, F}$ \\ 1 Department of Anesthesiology and Intensive Therapy, Pomeranian Medical University, Szczecin, Poland \\ ${ }^{2}$ Department of Medical Chemistry, Pomeranian Medical University, Szczecin, Poland \\ ${ }^{3}$ Department of Anesthesiology, Intensive Therapy and Acute Intoxications, Pomeranian Medical University, Szczecin, Poland \\ ${ }^{4}$ Department of Human Nutrition, West Pomeranian University of Technology, Szczecin, Poland \\ ${ }^{5}$ Department of Biochemistry and Chemistry, Pomeranian Medical University, Szczecin, Poland \\ A - research concept and design; $B$ - collection and/or assembly of data; $C$ - data analysis and interpretation; \\ $D$ - writing the article; $E$ - critical revision of the article; $F$ - final approval of the article
}

Address for correspondence

Edyta Barnik

E-mail: edytabarnik@op.pl

\section{Funding sources}

None declared

\section{Conflict of interest}

None declared

Received on December 8, 2016

Reviewed on January 5, 2017

Accepted on June 26, 2017

\begin{abstract}
Background. Hemolysis during cardiopulmonary bypass may lead to acute kidney injury caused by an excessive amount of iron. The clinical usefulness of the measurement of total iron concentration in the urine with the use of the atomic absorption spectrometry method for early identification of patients with postoperative acute kidney injury is not well-established.

Objectives. An observational, prospective study was conducted on a group of 88 pre-selected adult patients undergoing a planned coronary artery bypass grafting (CABG) procedure.

Material and methods. The amount and concentrations of total iron, creatinine and neutrophil gelatinaseassociated lipocalin (NGAL) were evaluated in urine samples. A comparative analysis of the evaluated biochemical parameters was performed in regard to the occurrence of acute kidney injury $48 \mathrm{~h}$ postoperatively.

Results. Patients in the acute kidney injury group presented more advanced age ( $p=0.01)$, preoperative myocardial infarction $(p=0.02)$, diuresis reduction $(p=0.04)$, and lower total iron levels in the 48-hour urine sample $(p=0.01)$. There was no difference when considering iron concentration in single urine samples in the study group.

Conclusions. The sole result of total iron concentration in single urine samples is unreliable for the diagnosis of acute kidney injury after cardiac surgery. Decreased excretion of iron in the urine seems to be an important additional element in the multifactorial pathogenesis of acute postoperative kidney failure.
\end{abstract}

Key words: iron, acute kidney injury, atomic absorption spectrometry

DOI

10.17219/acem/75504

Copyright

Copyright by Author(s)

This is an article distributed under the terms of the

Creative Commons Attribution Non-Commercial License

(http://creativecommons.org/licenses/by-nc-nd/4.0/) 
Cardiac surgery-associated acute kidney injury (CSA-AKI) is an important clinical problem. Kidney injury usually occurs following a cardiac surgery procedure. ${ }^{1-3}$ The pathomechanism of acute kidney injury (AKI) after cardiac surgery procedures performed with cardiopulmonary bypass (CPB) is complex and multifactorial. It involves several injury pathways: endogenous and exogenous toxins, ischemia and reperfusion, metabolic factors, inflammation, and oxidative stress. ${ }^{4,5}$

Undoubtedly, an important and frequently underlined factor increasing the negative effect of cardiopulmonary bypass on kidney function is oxidative stress. ${ }^{6-9}$ The generation of free oxygen species is catalyzed by free iron ions (Haber-Weiss and Fenton reactions), which are especially active in acidic environments. ${ }^{10}$ It has been shown in experimental studies that strategies targeting iron toxicity and renal protection using iron-chelating agents have a positive effect on pigment nephropathy and acute myocardial infarction. ${ }^{11,12}$ Until recently, there has been no official recommendations toward their routine use.

It is a fact that nephron overload due to an excessive amount of iron released during hemolysis initiates a vicious cycle, where progressive oliguria makes it impossible to excrete excess iron, thus leading to a prolongation of the toxic effect of iron. On the other hand, there is some data indicating that urine iron is bound by urine neutrophil gelatinase-associated lipocalin (NGAL), or if unbound, could be reabsorbed at the Henle's loop. ${ }^{13}$ Akrawinthawong et al. reported the clinical usefulness of urine (not plasma) iron concentration in a pilot study. They suggested that there is an overspill mechanism from hemolysis combined with overwhelming iron reabsorption at the tubules and collecting ducts and decreased reabsorption of iron in the tubules as AKI develops. The authors concluded that baseline and postoperative serial urine catalytic iron measurements are valuable indicators of AKI after open heart surgery. ${ }^{13}$ A question arises of whether perioperative determination of total iron level in the urine could serve as a good marker for the diagnosis of CSA-AKI. To date, there has been no definitive answer to that question. Therefore, any additional element helpful in the understanding of reasons for postoperative kidney failure enables the establishment of proper therapeutic procedures.

This research paper assessed the clinical utility of the determination of total iron level in the urine, using atomic absorption spectrometry for the identification of patients with postoperative AKI. Our study was concentrated on assessing the utility of urine iron at $1 \mathrm{~h}$ postoperatively in the early AKI detection. We also analyzed the dynamism of urine iron level changes within the first $24 \mathrm{~h}$ after a cardiac procedure.

\section{Material and methods}

This observational, prospective study was conducted in a group of 88 Caucasian patients over the age of 18 years after signing an informed consent form.
All patients underwent a planned procedure of cardiac artery bypass grafting (CABG) with the use of $\mathrm{CPB}$ in the Department of Cardiac Surgery of the Pomeranian Medical University in Szczecin (Poland). The study protocol was approved by the local Ethical Committee of the Pomeranian Medical University in Szczecin, Poland (KB - 0012/146/10).

The inclusion criteria of the study covered a planned operation of CABG with the use of CPB. Exclusion criteria regarding the preoperative period were defined as:

- emergency operations or re-operations;

- a known pathology of the urinary tract or renal failure;

- chronic use of the following medications: iron, nonsteroidal anti-inflammatory drugs (NSAIDs), immunosuppression, or steroids in the preoperative period;

- polycythemia, porphyria or pathological hemoglobin species in anamnesis;

- preoperative signs of hepatic failure;

- active autoimmune or neoplastic diseases, active infection;

- anticipated significant bleeding (anti-platelet agents), suggesting the use of blood-derived products during the operation and afterward.

The study population was divided into 2 groups regarding postoperative AKI: group "non-AKI" ( $=75)$, aged $62 \pm 7$ years, 61 men $(81 \%)$ and group "AKI" $(n=13)$, aged $70 \pm 6$ years, 9 men (69\%).

Acute kidney injury was defined according to Acute Kidney Injury Network (AKIN) criteria. $^{3}$

Before the cardiac operation, full physical examinations were performed to assess the patients' clinical status and to qualify them for the study. In the operating room, standard patient monitoring was initiated and general anesthesia was induced using fentanyl, etomidat, pancuronium (doses calculated according to body weight), and sevoflurane. Electrocardiography (ECG), invasive blood pressure monitoring, central venous pressure, deep body temperature, and mechanical ventilation parameters were monitored continuously; diuresis and fluid balance were evaluated on an hourly basis, during the operation and for the first $24 \mathrm{~h}$ postoperatively, after that time, every $12 \mathrm{~h}$. Metabolic monitoring was performed with a blood gas and electrolyte analysis in arterial blood using a GEM 3000 machine (Instrumentation Laboratory, Bedford, USA). Plasma iron concentration was measured on the day of the operation. Iron was determined with an atomic absorption spectrometer which had been calibrated using standard solutions. Measurements were made in an air-acetylene flame against the corresponding lamps at a wavelength of $248.3 \mathrm{~nm}$.

Serum creatinine levels and other standard laboratory parameters were evaluated on the day of the operation and $24 \mathrm{~h}$ and $48 \mathrm{~h}$ postoperatively.

Additionally, we measured NGAL level in the urine using a commercially available enzyme-linked immunosorbent assay (ELISA) kit (Human Lipocalin-2/NGAL Quantikine 
ELISA Kit; R\&D Systems, Minneapolis, USA) with a microplate reader ELx808 (BIO-TEK Instruments, Inc., Winooski, USA), according to the manufacturer's instructions. The time-points for NGAL measurement were $1 \mathrm{~h}$ after CPB (post-op sample) and $24 \mathrm{~h}$ from the beginning of the operation (postoperative day 1, POD 1 sample).

Moreover, an analysis of total iron concentration in single urine samples was performed. The method for performing the biochemical studies is presented below. After collection, the urine was centrifuged $\left(1850 \mathrm{~g}, 10 \mathrm{~min}, 4^{\circ} \mathrm{C}\right)$. The supernatant was immediately frozen to $-80^{\circ} \mathrm{C}$ until the analysis was performed. Iron level in the urine was evaluated using mass atomic absorption spectrometry by means of an absorption meter (PU 9100X; Philips, Cambridge, England). The applied wavelength was $248.3 \mathrm{~nm}$. Without any intervention, the normal value for total iron in the 24-hour urine was set at 40-150 $\mu \mathrm{g}(0.71-2.68 \mu \mathrm{mol})$. The normal value for total iron in the 24-hour urine of cardiac surgery patients (after CPB as an intervention) is unknown. The time-points for urine total iron concentration measurement were preoperative (zero sample), $1 \mathrm{~h}$ after CPB (post-op sample), and over $24 \mathrm{~h}$ from the beginning of the operation (POD 1 sample). Plasma-free hemoglobin concentration was measured in a reaction with Drabkin's reagent. The change of absorbance was noticed at wavelengths of $540 \mathrm{~nm}$ and $680 \mathrm{~nm}$. The normal value is $5-40 \mathrm{mg} / \mathrm{dL}$.

Cardiopulmonary bypass was performed using a nonpulsatile pump (Maquet, Hirrlingen, Germany) and a membrane oxygenator (Terumo Cardiovascular Systems, Ann Arbor, USA) primed with 1000 mL of Ringer's lactate, $500 \mathrm{~mL}$ of Gelofusine, $60 \mathrm{mEq}$ of sodium bicarbonate, and $4 \mathrm{mg} / \mathrm{kg}$ of heparin. An individual CPB flow was calculated on the basis of $2.5 \mathrm{~L} / \mathrm{min} / \mathrm{m}^{2}$ and systemic arterial blood pressure was maintained between 50 and $70 \mathrm{~mm} \mathrm{Hg}$. If the systemic perfusion pressure decreased, CPB flow was increased up to a maximum of $130 \%$ of the calculated flow.

Table 1. Demographic data and comorbidities of the study population

\begin{tabular}{|c|c|c|c|}
\hline Characteristics & $\begin{array}{c}\text { Non-AKI } \\
n=75\end{array}$ & $\begin{array}{c}\text { AKI } \\
n=13\end{array}$ & $p$-value \\
\hline Age [years], mean $\pm S D$ & $62 \pm 7$ & $70 \pm 6$ & $<0.01$ \\
\hline $\mathrm{BMI}\left[\mathrm{kg} / \mathrm{m}^{2}\right]$, mean $\pm \mathrm{SD}$ & $28.02 \pm 3.71$ & $31.20 \pm 3.98$ & 0.01 \\
\hline LV EF [\%], mean \pm SD & $53.52 \pm 9.57$ & $46.85 \pm 12.79$ & 0.08 \\
\hline EuroScore [points], mean $\pm S D$ & $2.36 \pm 1.91$ & $3.77 \pm 2.62$ & 0.07 \\
\hline Gender [male], n [\%] & $61(81)$ & $9(69)$ & 0.32 \\
\hline Left ventricular dysfunction NYHA $\geq I I, n$ [\%] & $48(64)$ & $11(84)$ & 0.53 \\
\hline Myocardial infarction, $\mathrm{n}$ [\%] & $31(41)$ & $10(76)$ & 0.02 \\
\hline Arterial hypertension, $\mathrm{n}$ [\%] & $64(85)$ & $11(84)$ & 0.46 \\
\hline Diabetes mellitus, n [\%] & $23(30)$ & $8(61)$ & 0.07 \\
\hline Peripheral vascular disease, $\mathrm{n}$ [\%] & $74(98)$ & $13(100)$ & 0.33 \\
\hline Atrial fibrillation, n [\%] & $4(5)$ & $1(7)$ & 0.75 \\
\hline
\end{tabular}

AKI - acute kidney injury; BMI - body mass index; LV EF - left ventricle ejection fraction before procedure; EuroScore - European System for Cardiac Operative Risk Evaluation; NYHA - New York Heart Association.
If the systemic pressure decrease could not be compensated for by increasing CPB flow, a norepinephrine infusion was used. The operation was performed in normothermia.

Statistical analysis included evaluating the changes of serum creatinine concentration and the biochemical parameters in the urine (total iron and creatinine) in relation to the occurrence of AKI according to the AKIN criteria up to $48 \mathrm{~h}$ postoperatively.

\section{Statistical analysis}

All results are presented as mean \pm standard deviation (SD). The Shapiro-Wilk test was used to check for normality of the sample data. To determine the differences between the AKI group and the non-AKI group, the Mann-Whitney $\mathrm{U}$ test was used for quantitative variables and Pearson's $\mathrm{X}^{2}$ test was used for qualitative variables. A logistic regression model was performed to predict whether urine iron concentration at defined time-points is a postoperative AKI risk factor based on the patient's age (covariate). The independent variables were urine iron concentration and age. The analysis was done using STATISTICA v. 10 software (StatSoft, Kraków, Poland). Statistical significance was set at $\mathrm{p}<0.05$.

\section{Results}

Early postoperative kidney failure based on the AKIN criteria was diagnosed in 13 patients (14\%) during the first $48 \mathrm{~h}$ postoperatively. In all of the cases, the AKIN diuresis criteria were equivalent to the AKIN creatinine criteria. Cardiac surgery-associated acute kidney injury was diagnosed in 6 patients within the first $6 \mathrm{~h}$ postoperatively (AKIN stage 1, next progression to stage 2 based on oliguria) and in 7 patients within the 48-hour period after the procedure based on oliguria and serum creatinine level (AKIN stage 2). The characteristics of the patient population are shown in Table 1.

When comparing the time of $\mathrm{CPB}$, minimal mean arterial pressure, the need for vasopressors or inotropes, and minimal body temperature, there were no differences between the 2 subgroups in the intraoperative period. In the study group, none of the patients needed perioperative transfusion of blood products. Selected data from the intraoperative period and the first $24 \mathrm{~h}$ postoperatively are presented in Table 2 .

There were no statistically significant differences in the concentration of plasma iron or serum and urine creatinine, nor in the urinary iron concentration in the preoperative period (zero sample) or directly after the CPB (POD 1 sample). There were differences in diuresis in the intraoperative and postoperative periods, though. Therefore, the excretion of total iron was not only shown 
Table 2. Interventions and clinical outcomes of the study population

\begin{tabular}{|l|c|c|c|}
\multicolumn{1}{|c|}{ Characteristics (mean \pm SD) } & $\begin{array}{c}\text { Non-AKI } \\
n=75\end{array}$ & $\begin{array}{c}\text { AKI } \\
n=13\end{array}$ & p-value \\
\hline \multicolumn{1}{|c|}{ Interventions } & $48 \pm 13$ & $48 \pm 15$ & 0.78 \\
\hline Cardiopulmonary bypass time [min] & $29 \pm 7$ & $29 \pm 8$ & 0.71 \\
\hline Aorta cross-clamp time [min] & $167 \pm 28$ & $165 \pm 24$ & 0.82 \\
\hline Surgical procedure time [min] & $68 \pm 8$ & $64 \pm 6$ & 0.24 \\
\hline Minimal value of MAP (CPB) [mm Hg] & $659 \pm 389$ & $453 \pm 368$ & 0.04 \\
\hline Urine output during a surgical procedure [mL] & $715 \pm 569$ & $530 \pm 765$ & 0.33 \\
\hline Fluid balance during a surgical procedure $[\mathrm{mL}]$ & $3215 \pm 799$ & $2607 \pm 460$ & $<0.01$ \\
\hline Urine output [mL] ${ }^{*}$ & $-627 \pm 1204$ & $-584 \pm 780$ & 0.87 \\
\hline Fluid balance [mL]
\end{tabular}

AKI - acute kidney injury; MAP - mean arterial pressure; CPB - cardiopulmonary bypass; $\mathrm{SD}$ - standard deviation; ${ }^{*}$ from the beginning of the operation to $24 \mathrm{~h}$ thereafter.

\section{Discussion}

The pathomechanism of pigment nephropathy after cardiac surgery is based on kidney injury secondary to the influence of free hemoglobin released from erythrocytes during CPB. ${ }^{7}$ Active free iron ions are generated during erythrocyte damage and hemolysis by the $\mathrm{CPB}$ set. A sudden increase in the amount of iron can exceed the iron binding capacity. As a result, it can be observed that many changes occur within the epithelium of the tubular cells. Previous studies confirmed iron toxicity in the case of acute and chronic kidney failure, acute cardiac ischemic injury and neurodegenerative diseases. ${ }^{8-10,14,15}$ There is evidence from

as the concentration in a single sample, but also as the total amount of iron in the urine collected $24 \mathrm{~h}$ postoperatively. A comparison of creatinine, total iron and NGAL excretion in the urine in pre-defined time-points is shown in Table 3.

We compared the changes of free hemoglobin (plasma) concentration in 65 patients (no-AKI group) and 13 patients (AKI group) at defined time-points. There were no significant differences.

The logistic regression model showed that the patient's age (covariate) is not a factor determining postoperative AKI. This result was observed in all variables in the study.

Table 3. Perioperative values of serum and urine parameters in the study population

\begin{tabular}{|c|c|c|c|}
\hline Characteristics (mean $\pm \mathrm{SD}$ ) & $\begin{array}{c}\text { Non-AKI } \\
n=75\end{array}$ & $\begin{array}{c}\text { AKI } \\
n=13\end{array}$ & $\mathrm{p}$-value \\
\hline \multicolumn{4}{|c|}{ Preoperative (zero sample) } \\
\hline Iron concentration [mg/L $\left.\mathrm{L}^{-1}\right]$ (urine) & $\begin{array}{c}0.12 \pm 0.06 \\
\text { OR }=4.5 \times 10^{-5} \\
95 \% \mathrm{Cl}: 8.15 \times 10^{-10}-2.5\end{array}$ & $\begin{array}{c}0.14 \pm 0.07 \\
0.07\end{array}$ & 0.07 \\
\hline Iron concentration $\left[\mathrm{mg} / \mathrm{dL}^{-1}\right]$ (plasma) & $130.8 \pm 27.21$ & $123.9 \pm 6.1$ & 0.92 \\
\hline Creatinine $\left[\mathrm{mg} / \mathrm{dL}^{-1}\right]$ (serum) & $0.88 \pm 0.19$ & $0.96 \pm 0.28$ & 0.47 \\
\hline \multicolumn{4}{|c|}{$1 \mathrm{~h}$ after the end of CPB (post-op sample) } \\
\hline Iron concentration [mg/L $\left.\mathrm{L}^{-1}\right]$ (urine) & $\begin{array}{c}0.08 \pm 0.05 \\
\mathrm{OR}=9.3 \times 10^{-4} \\
95 \% \mathrm{Cl}: 6.28 \times 10^{-9}-138.9\end{array}$ & $\begin{array}{c}0.10 \pm 0.06 \\
0.24\end{array}$ & 0.29 \\
\hline $\mathrm{NGAL}\left[\mathrm{ng} / \mathrm{mL}^{-1}\right]$ (urine) & $3.12 \pm 7.20$ & $9.30 \pm 13.78$ & $<0.01$ \\
\hline \multicolumn{4}{|c|}{$24 \mathrm{~h}$ after the beginning of the operation (POD 1 sample) } \\
\hline Iron concentration $\left[\mathrm{mg} / \mathrm{L}^{-1}\right]$ (urine) & $\begin{array}{c}0.12 \pm 0.05 \\
\text { OR }=320.74 \\
95 \% \text { Cl: } 5.58 \times 10^{-4}-1.8 \times 10^{4}\end{array}$ & $\begin{array}{c}0.09 \pm 0.06 \\
0.38\end{array}$ & 0.09 \\
\hline Total iron (urine) ${ }^{*}$ & $407 \pm 191.01$ & $268 \pm 120.74$ & $<0.01$ \\
\hline Creatinine $\left[\mathrm{mg} / \mathrm{dL}^{-1}\right]$ (serum) & $0.86 \pm 0.22$ & $1.29 \pm 0.38$ & $<0.01$ \\
\hline NGAL [ng/mL $\left.L^{-1}\right]$ (urine) & $12.19 \pm 11.76$ & $25.02 \pm 11.89$ & $<0.01$ \\
\hline \multicolumn{4}{|c|}{$48 \mathrm{~h}$ after the beginning of the operation (POD 2 sample) } \\
\hline Creatinine $\left[\mathrm{mg} / \mathrm{dL}^{-1}\right]$ (serum) & $0.84 \pm 0.25$ & $1.67 \pm 0.55$ & $<0.01$ \\
\hline
\end{tabular}

AKI - acute kidney injury; CPB - cardiopulmonary bypass; POD - postoperative day; NGAL - neutrophil gelatinase-associated lipocalin; OR - odds ratio; Cl - confidence interval; SD - standard deviation; * the mean \pm SD of total iron ( $\mu \mathrm{g} /$ day) excreted in the urine during the $1^{\text {st }}$ postoperative day (urine collected for $24 \mathrm{~h}$ ). animal studies that iron is involved in a variety of models of AKI. However, there is limited data from human studies to support our study.

An important human defense mechanism in the case of iron overload is iron excretion in the urine. One can imagine that the assessment of iron excretion in the urine would be an ideal marker of this pathomechanism. However, one must take into account the fact that a reduction of diuresis and increased NGAL concentration (an important iron-translocating compound) can modify final iron excretion. The result of our study was that patients with AKI showed significant differences in total iron levels in the urine collected $24 \mathrm{~h}$ postoperatively, as assessed by atomic absorption spectrometry. Urine iron level was assessed in the first $24 \mathrm{~h}$ only because we defined this as an early marker. In our opinion, a decrease of urine output as a clinical manifestation of AKI might cause a decrease in the iron-excreting ability in the AKI group. This fact underlines the importance of actual diuresis and fluid balance when the purifying function of the kidneys is analyzed.

In our study, total iron excreted with the urine was elevated in all patients in the study group. However, to date no validated "normal values" for cardiac surgery patients have been determined. The most important conclusion of this study is that the single result of total iron concentration in a single urine sample is unreliable for AKI diagnosis after cardiac 
surgery. This result is opposite to the findings of the sole human pilot study. ${ }^{13}$ The authors of that trial noticed increased levels of urine catalytic iron at the same time as urine NGAL. In our opinion, this difference is caused by the very small sample size. In our unpublished data, we observed a similar trend in the first few results. In the current study, we observed a rise of urine NGAL at $1 \mathrm{~h}$ and $24 \mathrm{~h}$ postoperatively. However, when we considered the values of serum creatinine levels and urine NGAL levels in individual patients, we detected some differences. Regarding serum creatinine, we diagnosed 13 AKI cases within the first $48 \mathrm{~h}$ after operation. However, an elevated NGAL level was observed in 5 of 13 patients and in 4 cases without AKI. To date, NGAL is not a criterion in the recognizing and staging of AKI. Akrawinthawong et al. showed the results of serial urine catalytic iron measurements, but urine catalytic iron and NGAL were first measured $8 \mathrm{~h}$ postoperatively. We cannot compare our results because in our study the time-point was different ( $1 \mathrm{~h}$ after the end of $\mathrm{CPB}){ }^{13}$

A decrease in diuresis is a clinical manifestation of renal tubule insufficiency. This fact underlines the need for the purifying function of the kidneys in the context of actual diuresis and fluid balance, as the changes in the amount of iron are usually minimal and the clinical implications are very important. Acute kidney injury occurred more frequently in patients with a past medical history of myocardial infarction. This fact could be explained as follows: oxygen free radicals are generated as a consequence of oxidative stress related to myocardial infarction and this process is catalyzed by iron ions. These free radicals cause organ damage, including the kidneys. The presence of another damaging factor, i.e., $\mathrm{CPB}$, leads to an increase of the previously subclinical kidney damage.

\section{Iron measurement methods}

The question is: what should we measure - plasma or urine iron? Leaf et al. showed that increased plasma catalytic iron in patients might mediate AKI and death following cardiac surgery. ${ }^{16}$ However, plasma catalytic iron concentration may increase due to reduced filtration. In the study by Leaf et al., urine output was not assessed. They did not use oliguria as an AKI criterion. In the available literature, the measurement of urine output regarding its purifying role is crucial. The amount of urine output during CPB can be a simple method to predict the development of AKI after cardiac surgery. ${ }^{17}$ The population analyzed by Leaf et al. was heterogenic (the majority of patients had valve or mixed procedures). More than $30 \%$ of the procedures were urgent or re-operations. The mean $\mathrm{CPB}$ time was 3 -fold longer than in our study. All these factors are important regarding hemolysis, load of iron, the staging of cardiac surgery-related AKI, and the need for renal replacement therapy in the case of postoperative AKI. However, the authors did not show a comparison among the group regarding these factors, so we cannot compare results to our "isolated CABG group." In our study, the population is homogenic. The variation in urine iron excretion is also noticeable, but statistically insignificant among the study group.

The role of iron as a mediator of CSA-AKI is still not fully appreciated; therefore, the methods of iron measurement are not widely used in clinical practice. The chemical identification of the labile iron pool is difficult due to the variety of iron ligands present in cells. ${ }^{10}$ Atomic absorption spectrometry is an analysis technique which enables iron determination in liquid, solid and gas samples. The analysis is based on the absorption of radiation at a specific wavelength by free iron atoms, evaluation of its absorbency, and therefore its ion concentration. This method enables the detection of even minimal quantities of the metal in a sample. This is especially important in patients after CABG, because perioperative hemodilution leads to a situation where the amount of iron in the analyzed sample is minimal. The clinical utility of this method and the indications for its routine use require further studies.

One source of iron is hemolysis. Hemolysis is a recognized consequence of $\mathrm{CPB}$. It has been shown to increase with complex procedures (CABG + valve surgery) because of longer perfusion times. ${ }^{18}$ Hemolysis during cardiac surgery may be diagnosed and monitored by plasma extracellular (free) hemoglobin and plasma haptoglobin (the physiological intravascular free hemoglobin scavenger) concentration, assessed at regular time-points. However, there were no significant differences in our results.

One limitation of the current study is the lack of plasma iron concentrations at $1 \mathrm{~h}$ and $24 \mathrm{~h}$ postoperatively. Another limitation is the lack of plasma and urinary iron measurements $48 \mathrm{~h}$ postoperatively.

In conclusion, the presented study confirms that the sole result of total iron concentration in a single urine sample is unreliable in the diagnosis of AKI after cardiac surgery. This study indicates that decreased iron excretion with decreased urine volume seems to be an important additional element in the multifactorial pathogenesis of acute postoperative kidney failure. It is possible that in the future, iron-chelating compounds or elective intraoperative hemofiltration will be used as a method of targeted preventive therapy. In the early postoperative period, the interpretation of biochemical parameters in the context of current diuresis and fluid balance seems to be of critical importance.

\section{References}

1. Bellomo R, Auriemma S, Fabbri A, et al. The pathophysiology of cardiac surgery-associated acute kidney injury (CSA-AKI). Int J ArtifOrgans. 2008;31:166-178.

2. McCullough PA, Kellum JA, Haase M, et al. Pathophysiology of the cardiorenal syndromes: Executive summary from the eleventh consensus conference of the Acute Dialysis Quality Initiative (ADQI). Contrib Nephrol. 2013;182:82-98.

3. Mehta RL, Kellum JA, Shah SV, et al. Acute Kidney Injury Network: report of an initiative to improve outcomes in acute kidney injury. Crit Care. 2007;11:R31. doi: 10.1186/cc5713 
4. O'Neal J, Shaw A, Billings F. Acute kidney injury following cardiac surgery: Current understanding and future directions. Crit Care. 2016;20:187. doi: 10.1186/s13054-016-1352-z

5. Haase-Fielitz A, Bellomo R, Devarajan P, et al. Novel and conventional serum biomarkers predicting acute kidney injury in adult cardiac surgery - a prospective cohort study. Crit Care Med. 2009;37:553-560.

6. Mao H, Katz N, Ariyanon W. Cardiac surgery-associated acute kidney injury. Cardiorenal Med. 2013;3:178-199. doi: 10.1159/000353134

7. Haase M, Haase-Fielitz A, Bagshaw SM, Ronco C, Bellomo R. Cardiopulmonary bypass-associated acute kidney injury: A pigment nephropathy? Contrib Nephrol. 2007;156:340-353.

8. Shah S, Rajapurkar M, Baliga R. The role of catalytic iron in acute kidney injury. Clin J Am Soc Nephrol. 2011;6:2329-2331.

9. Haase M, Bellomo R, Haase-Fielitz A. Novel biomarkers, oxidative stress, and the role of labile iron toxicity in cardiopulmonary bypassassociated acute kidney injury. J Am Coll Cardiol. 2010;55:2024-2033.

10. Kakhlon O, Cabantchik ZI. The labile iron pool: Characterization, measurement, and participation in cellular processes. Free Radic Biol Med. 2002;33:1037-1046.

11. Ambrus CM, Stadler I, Toumbis CA, et al. Removal of non-transferrin-bound iron from blood with iron overload using a device with immobilized desferrioxamine. J Med. 1999;30:211-224.
12. Paraskevaidis IA, lliodromitis EK, Vlahakos D, et al. Deferoxamine infusion during coronary artery bypass grafting ameliorates lipid peroxidation and protects the myocardium against reperfusion injury: Immediate and long-term significance. Eur Heart J. 2005;26:263-270.

13. Akrawinthawong $K$, Shaw M, Kachner J, et al. Urine catalytic iron and neutrophil gelatinase-associated lipocalin as companion early markers of acute kidney injury after cardiac surgery: A prospective pilot study. Cardiorenal Med. 2013;3(1):7-16.

14. Korsak J. Posttransfusion iron overload. Pol Merk Lek. 2011;30:177.

15. Martines AM, Masereeuw R, Tjalsma H, Hoenderop JG, Wetzels JF, Swinkels DW. Iron metabolism in the pathogenesis of iron-induced kidney injury. Nat Rev Nephrol. 2013;9(7):385-398. doi: 10.1038/nrneph.2013.98

16. Leaf DE, Rajapurkar M, Lele SS, et al. Increased plasma catalytic iron in patients may mediate acute kidney injury and death following cardiac surgery. Kidney Int. 2015;87(5):1046-1054. doi: 10.1038/ki.2014.374

17. Song Y, Kim DW, Kwak YL. Urine output during cardiopulmonary bypass predicts acute kidney injury after cardiac surgery: A single center retrospective analysis. Medicine (Baltimore). 2016;95(22):e3757.

18. Vermeulen Windsant IC, de Wit NC, Sertorio JT, et al. Hemolysis during cardiac surgery is associated with increased intravascular nitric oxide consumption and perioperative kidney and intestinal tissue damage. Front Physiol. 2014;8(5):340. doi: 10.3389/fphys.2014.00340 